\title{
The influence of global warming in Earth rotation speed
}

\author{
R. Abarca del Rio \\ LEGOS-UMR5566-GRGS, 18 Av. Ed Belin, F-31400 Toulouse, France, e-mail: Rodrigo.Abarca-del-Rio@cnes.fr
}

Received: 7 December 1998 / Revised: 21 January 1999 / Accepted: 29 January 1999

\begin{abstract}
The tendency of the atmospheric angular momentum (AAM) is investigated using a 49-year set of monthly AAM data for the period January 1949December 1997. This data set is constructed with zonal wind values from the reanalyses of NCEP/NCAR, used in conjunction with a variety of operationally produced AAM time series with different independent sources and lengths over 1976-1997. In all the analyzed AAM series the linear trend is found to be positive. Since the angular momentum of the atmosphere-earth system is conserved this corresponds to a net loss of angular momentum by the solid earth, therefore decreasing the Earth rotation speed and increasing the length of day (LOD). The AAM rise is significant to the budget of angular momentum of the global atmosphere-earth system; its value in milliseconds/century (ms/cy) is $+0.56 \mathrm{~ms} / \mathrm{cy}$, corresponding to one-third of the estimated increase in LOD $(+1.7 \mathrm{~ms} / \mathrm{cy})$. The major contribution to this secular trend in AAM comes from the equatorial Tropopause. This is consistent with results from a previous study using a simplified aqua-planet model to investigate the AAM variations due to near equatorial warming conditions. During the same time interval, 1949-1997, the global marine + land-surface temperature increases by about $0.79{ }^{\circ} \mathrm{C} / \mathrm{cy}$, showing a linear correspondence between surface temperature increase and global AAM of about $0.07 \mathrm{~ms}$ per $0.1{ }^{\circ} \mathrm{C}$. These results imply that atmospheric angular momentum may be used as an independent index of the global atmosphere's dynamical response to the greenhouse forcing, and as such, the length of day may be used as an indirect indicator of global warming.
\end{abstract}

Key words. Meteorology and atmospheric dynamics (general circulation), Geodesy

\section{Introduction and motivation}

A distinguishing characteristic of the Earth's rotation is that the rate of rotation varies with time. Changes in the length of the mean solar day (LOD) are used to describe the changes in the rotation rate. Analyzing observations of eclipses dating back 2500 years, Stephenson and Morrison (1995) showed that the LOD has increased on average by $+1.7 \pm 0.05$ milliseconds/century (ms/cy). The astronomical evidence points to a secular change in the mean motion of the moon. With the requirement that angular momentum must be conserved, this observation means that the Earth must also be subject to a tidal acceleration. The acceleration of the moon, measured accurately from lunar laser ranging and from the observed perturbations on the orbits of artificial satellites causes by lunar tides (Christodoulis et al., 1988), allowed Stephenson and Morrison (1995) to compute the corresponding change in LOD as $+2.3 \pm 0.1 \mathrm{~ms} / \mathrm{cy}$. Thus, there must be an average accelerative non tidal component in the Earth's rotation which acts to decrease the LOD by $-0.6 \pm 0.1 \mathrm{~ms} / \mathrm{cy}$. This is commonly explained by the effects of the last ice age (Wahr, 1988); the postglacial rebound induces a net transfer of material within the Earth toward higher northern latitudes, decreasing the Earth's moment of inertia and thereby decreasing LOD. Using laserranging data from Lageos and Starlette satellites, it has been possible to measure changes in the Earth's gravitational field and estimate secular variability in LOD due to internal mass distribution within the Earth system. These measurements range from $-0.44 \pm 0.05 \mathrm{~ms} / \mathrm{cy}$ to $-0.61 \pm 0.05 \mathrm{~ms} / \mathrm{cy}$, consistent with values we should expect from a postglacial rebound process (Peltier and Jiang, 1996).

The LOD signal also undergoes diurnal to decadal variability (Lambeck, 1980). These fluctuations are of considerable geophysical interest since they imply that changes occur in the mass distribution inside and on the 
surface of the Earth, or that torques are applied at the Earth surface or at the core-mantle boundary (Lambeck, 1988). It has now been proven that changes in the axial momentum of the atmosphere's angular momentum (AAM) explains most of the changes in the length of the day (LOD) over the broad band range of time scales from diurnal up to the interannual (Eubanks, 1993). However on decadal and longer time-scales, nonatmospheric processes must dominate the global momentum budget; a view which has been appreciated since at least as long ago as Munk and MacDonald (1960).

Recently, Satoh and Yoshida (1996) applied an atmospheric aqua planet model to show that global uniform warming of surface temperature results in an increase of the AAM. These results, based on a simplified model were too weak to explain the decadal variability in LOD. However their ideas were supported by Abarca del Rio (1997), who used information on zonal winds from rawinsonde measurements and operational centers from 1963 to 1994 to show that part of the decadal variability on LOD could be excited by the atmosphere. Thus, in contradiction to Munk and MacDonald (1960), it is possible that long term atmospheric variability may be contributing to secular variability in LOD.

Many authors have studied long-term instrumental temperature records to separate possible anthropogenic climate change from natural climate variability (Nicholls et al., 1996 and references therein). All these studies concluded that a significant, but irregular warming trend has occurred on a global scale since 1850: global surface temperatures have increased by about $0.3-0.6{ }^{\circ} \mathrm{C}$ since the late nineteenth century, and by about $0.2-0.3{ }^{\circ} \mathrm{C}$ over the last 40 years (the period with the most credible data). The trend is fairly flat from the middle of the nineteenth century until 1910 , followed by increases of over $0.1{ }^{\circ} \mathrm{C}$ per decade in combined landand sea-surface temperature during 1910-1940 and over the last four decades. The short instrumental climate records at our disposal make it difficult to explain this irregular increase. This could be due to natural variability, external forcing such a slow insolation variations or changes in the mean terrestrial volcanism. However the most popular suspected cause is anthropogenic greenhouse gases which give rise to internal climate variability. This last hypothesis implies a linear increase in surface temperature (land and sea) given an exponential increase in carbon dioxide. This increase in global surface temperature, whatever its cause should be, may then possibly generate a trend in AAM.

The purpose of this paper is to investigate a trend in AAM, the relative contribution from the whole atmosphere as its possible relationship with surface temperature. This study is made possible thanks to the recent reanalyses of long records of climate variables by NCEP/NCAR (Kalnay et al., 1996) that provide zonal winds for almost 50 years (1949-1997). Information from independent AAM series derived from operational centers (NCEP, JMA, UKMO and ECMWF) is provided by the Sub Bureau of Atmospheres (SBA)
(Salstein et al., 1993) of the International Earth Rotation Service (IERS) which allows us to verify the reality of the estimates of the secular trend.

\section{Trend in AAM series}

The AAM about the polar axis of a layer of the atmosphere, may calculated by (Barnes et al., 1983)

$\mathrm{AAM}=\frac{2 \pi R^{3}}{g} \int_{P S}^{P t} \int_{\pi / 2}^{-\pi / 2} \int_{0}^{2 \pi} u \cos ^{2} \varphi \partial \lambda \partial \varphi \partial p$

where $R$ is the radius of the Earth, $g$ the acceleration due to gravity and $u$ denotes zonal wind. The term is integrated over all latitudes, longitudes and pressures $(\varphi, \lambda$ and $p)$. Because the wind fields are archived at constant pressures levels, it is convenient when using these data sets to set the lower boundary in the integration $\left(p_{S}\right)$ to $1000 \mathrm{hPa}$, whereas the upper level $\left(p_{T}\right)$ varies here from $100-10 \mathrm{hPa}$ depending on the operational weather center considered. All the characteristics of AAM series used in that study are summarized in Table 1. The longest series of values of operational AAM data already available from SBA are the $\mathrm{NMC}_{\mathrm{W}}^{100}$, integrated up to $100 \mathrm{hPa}$ produced by the NMC, now NCEP, from June 1976 to January 1997. Also starting in 1976 are the $\mathrm{NMC}_{\mathrm{W}}^{50}(50 \mathrm{hPa})$ series from NMC analyses. The $\mathrm{UK}_{\mathrm{W}}^{25}(25 \mathrm{hPa})$ series begins in May 1983 from the analyses of the United Kingdom Meteorological Office (UKMO). A series of values integrated up to $10 \mathrm{hPa}\left(\mathrm{EC}_{\mathrm{W}}^{10}\right)$ became available from the European Centre for Medium Range Weather Forecasts (ECMWF) starting in October 1984. Starting in september 1983, the Japan Meteorological Agency (JMA) also provides the $\mathrm{JMA}_{\mathrm{W}}^{10}$ series up to $10 \mathrm{mb}$. All operational AAM series extend up to January 1997. In summary, all these series collected and archived by the SBA (Salstein et al., 1993) are at least 10-years long. However, since decadal scale variability is also present

Table 1. Estimates of rates. AAM values are given in $10^{-3} \mathrm{~s}(\mathrm{~ms})$ and Temperatures in degrees Celsius $\left({ }^{\circ} \mathrm{C}\right)$. First column gives information on the AAM series; source, name, upper height integrated and length

\begin{tabular}{ll}
\hline Operational AAM series & Trend \\
\hline & $\mathrm{ms} / \mathrm{cy}$ \\
$\mathrm{JMA} \mathrm{JMA}$ & 0.59 \\
$\mathrm{ECMWF} \mathrm{EC}_{\mathrm{W}}^{10} 10 \mathrm{hPa}(83-97)$ & 0.48 \\
$\mathrm{UKMO}(84-97)$ & 0.70 \\
$\mathrm{NMC} \mathrm{NMC}_{\mathrm{W}}^{50} 25 \mathrm{hPa}(83-97)$ & 0.51 \\
$\mathrm{NMC} \mathrm{NMC}_{\mathrm{W}}^{100} 100 \mathrm{hPa}(76-97)$ & 0.39 \\
Reanalyses AAM series & \\
Reanalyses REA 10 & 0.56 \\
North hemisphere & 0.38 \\
South hemisphere & 0.17 \\
Surface temperature & ${ }^{\circ} \mathrm{C} / \mathrm{cy}$ \\
Land + ocean (49-97) & 0.79 \\
\hline
\end{tabular}


in AAM (Abarca del Rio, 1997), the determination of a long-term gradient in AAM can be biased by this or other low frequency signals present in the time series. The availability of zonal wind fields from the recent NCEP reanalyses (Kalnay et al., 1996) allows us to construct a 49-year-long AAM time series (January 1949-December 1997), that permits a better determination of decadal variability and thus a more accurate long-term trend. The reanalyses fields of NCAR/UCAR are available on a monthly basis on $2.5 \times 2.5$ latitudelongitude grids over 17 layers $(1000,925,850,700,600$, $500,400,300,250,200,150,100,70,50,30,20$ and $10 \mathrm{hPa}$ ). These fields allowed computation of AAM up to $10 \mathrm{hPa} \mathrm{REA}_{\mathrm{W}}^{10}$ using Eq. 1.

It is possible to infer changes in LOD from global AAM time series, assuming that changes in AAM for the entire atmosphere are accompanied by equal, but opposite, changes in the angular momentum of the earth, through the relation (Rosen and Salstein, 1983)

$\Delta \mathrm{LOD}(\mathrm{ms})=1.6810^{-29} \Delta \mathrm{AAM}\left(\mathrm{kg} \mathrm{m}^{2} / \mathrm{s}\right)$

where $\triangle \mathrm{LOD}$ is given in milliseconds. In this study all AAM series will be expressed in their equivalent value in LOD.

The REA $\mathrm{W}_{\mathrm{W}}^{10}$ series over 1949-1997 is shown in Fig. 1, together with its calculated linear trend. The trend is equal to $0.56 \mathrm{~ms} / \mathrm{cy}$. As an external check, we also estimate trends in the operational AAM series. Results for all the calculated trends are reported in Table 1. The estimated trends range from $0.48 \mathrm{~ms} / \mathrm{cy}$ for the $\mathrm{EC}_{\mathrm{W}}^{10}$ series to $0.70 \mathrm{~ms} /$ cy for the $\mathrm{UK}_{\mathrm{W}}^{25}$ series. Note that values from $\mathrm{NMC}_{\mathrm{W}}^{100}$ and $\mathrm{NMC}_{\mathrm{W}}^{50}$ are close, which demonstrate that the main contribution is from the troposphere $(79 \%)$; however the difference suggests that the lower stratosphere contributes significantly to this trend.

\section{Temperature changes and global AAM}

Over the same time span, the global (land + marine) temperature data set from the Climate Research Unit of

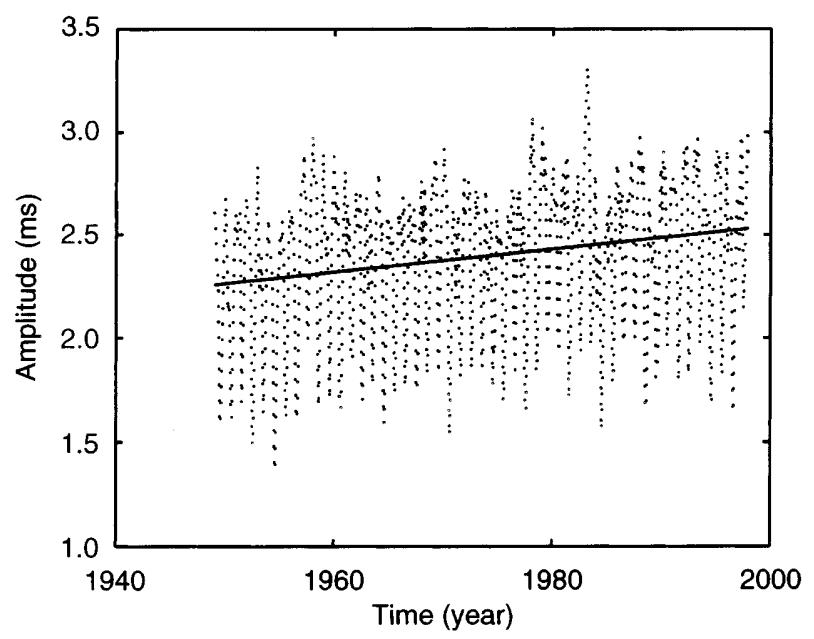

Fig. 1. Time series of AAM from 1949 to 1997 (points) and the estimated trend (solid) the University of East Anglia (Parker et al., 1994), increases by about $0.79^{\circ} \mathrm{C} / \mathrm{cy}$ (Table 1). Thus, if at secular time scales a relation exists between global surface temperature increase and global AAM increase the ratio would be a $0.07 \mathrm{~ms}$ per $0.1{ }^{\circ} \mathrm{C}$ increase. Indeed a close relation at interannual time-scales has already been shown between temperature and AAM (Stefanick, 1982) by assuming that changes in subtropical winds are qualitatively consistent with tropical temperatures changes assuming geostrophic balance. This study suggests that a thermal wind argument was sufficient for explaining the amplitude of the zonal wind variations. More recently, a modeling study by Satoh and Yoshida (1996) provided a first insight into a possible relation between increase in global temperature and AAM. Their results show that AAM increases when the temperature is uniformly increased due to the higher elevations of the Tropopause and the jets. Their study also demonstrated that when warming is confined near the equator, a jet is created within the equatorial Tropopause which leads to increased zonal winds and therefore to an important contribution to the global AAM trend. The mechanism by which the equatorial jet is created is related to Rossby wave generation in the equatorial area (Satoh, personal communication).

\section{Zonal and regional contributions to the AAM trend}

The monthly zonal wind fields from the NCEP reanalyses (1949-1997), were used to compute the relative contribution to the trend from all vertical layers from the surface $(1000 \mathrm{hPa})$ up to $10 \mathrm{hPa}$ on a global and hemispheric basis. For each hemisphere within each pressure level at which zonal wind data were available, we compute the angular momentum (Fig. 2). In order to stress the contribution from upper layers of the atmosphere, results in Figs. 2, 4 are presented according to height in kilometers $(\mathrm{km})$ instead of units of pressures (hPa). Indeed, a major contribution to the global AAM originates within the upper troposphere and lower stratosphere (10-26 km), with a broad peak about 15 $19 \mathrm{~km}$ (roughly 100-50 hPa) explaining results found with the $\mathrm{NMC}_{\mathrm{W}}^{100}$ and $\mathrm{NMC}_{\mathrm{W}}^{50}$ series. The vertical distribution in the northern hemisphere explains most of the prominent $10-25 \mathrm{~km}$ peak. A strong contribution to the global AAM is also found about $1-5 \mathrm{~km}$ (roughly 900-600 hPa) with a peak at $2.5 \mathrm{~km}$ (roughly $750 \mathrm{hPa}$ ) originated mainly from southern hemisphere contribution. Note that the southern hemisphere includes layers with negative contribution, located within the upper part of the Troposphere (10-15 km, roughly 200$100 \mathrm{hPa}$ ).

These hemispheric trends can be further examined by calculating the zonal contribution to the global AAM. Within each latitude width for which zonal wind data were available (every $2.5^{\circ}$ latitude) we compute the angular momentum (per unit of latitude width) from the surface $(1000 \mathrm{hPa})$ to the top $(10 \mathrm{hPa}$; Fig. 3). A negative contribution comes preferentially from the latitudes $45^{\circ} \mathrm{S}$ to $25^{\circ} \mathrm{S}$, which correspond to the position 


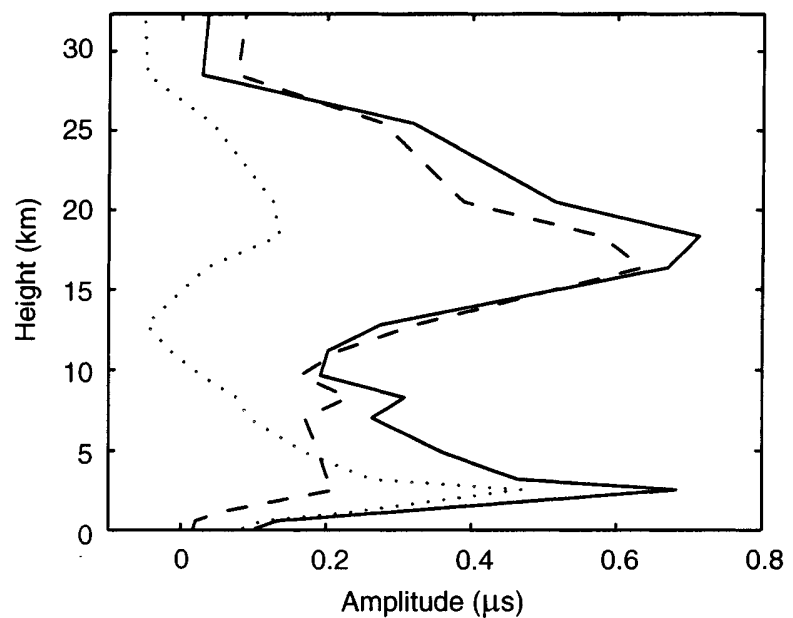

Fig. 2. Vertical distribution of contributions to the global AAM trend. Northern hemisphere (dashed), southern hemisphere (points) and sum of both contributions (solid). The ordinate is height in kilometers $(\mathrm{km})$. Values are given in units of $10^{-6} \mathrm{~s}$ (us)

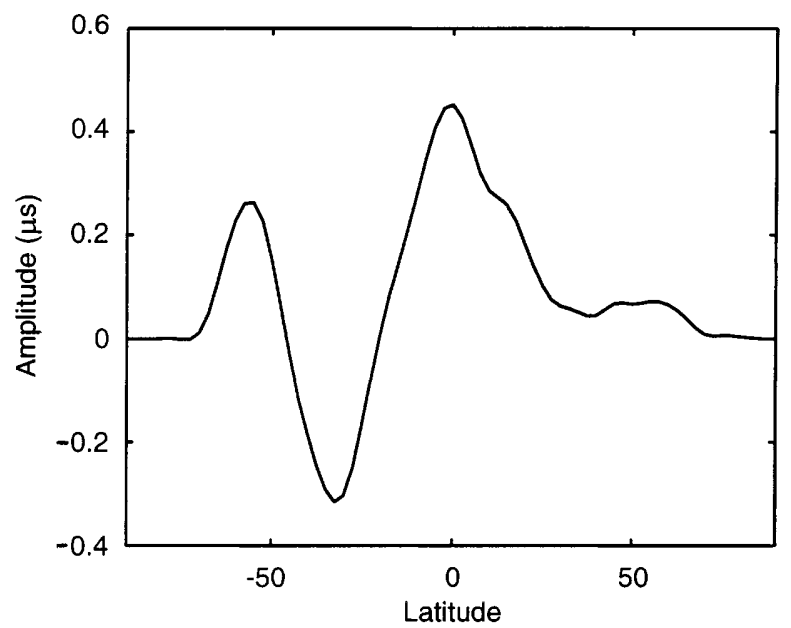

Fig. 3. Distribution of contributions over rings of $2.5^{\circ}$ latitude width to the global AAM trend. The ordinate is height in kilometers $(\mathrm{km})$. Values are given in units of $10^{-6} \mathrm{~s}$ (us)

of the southern hemisphere jet (Peixoto and Oort, 1992). Over the southern hemisphere a positive peak takes place from $70^{\circ} \mathrm{S}$ to $45^{\circ} \mathrm{S}$, with a maximum at about $56^{\circ} \mathrm{S}$, with only a very slight counterpart at the same northern latitude. However, the most important contribution to the global trend in AAM arises in tropical latitudes between $20^{\circ} \mathrm{S}$ to $20^{\circ} \mathrm{N}$. As the positive and negative contributions almost compensate each other in the southern hemisphere, the integrated value from $20^{\circ} \mathrm{S}$ to $20^{\circ} \mathrm{N}$ accounts for most of the trend found in the global AAM.

To give a further insight into which portions of the atmosphere are most responsible for the results found above, we estimated angular momentum within each latitude width (every $2.5^{\circ}$ ) and atmospheric layer for which zonal winds were available (Fig. 4). As already shown by Figs. 2 and 3, there is no symmetry with respect to the equator though similar properties are shown in both hemispheres. In both hemispheres strong negative trends find their source over the subtropical jets about $25^{\circ}-35^{\circ}$ latitudes, located within 10 and $15 \mathrm{~km}$ $(200-100 \mathrm{hPa})$ with a maximum of intensity around $30^{\circ}$ and $13 \mathrm{~km}(150 \mathrm{hPa})$. However, the altitudes of the negative trends are not exclusively located near the jets; they extend from the near surface $(800 \mathrm{mb})$ to the top of the atmosphere. The negatives trends correspond to the zone of exchange between the Hadley and Ferrel cells (Peixoto and Oort 92). Note that in the northern hemisphere the position of the jets is slightly equatorward than in southern hemisphere.

Strong positive contributions to the global AAM trend are found within the latitudes $50^{\circ}-60^{\circ}$ with maximum intensity at $55^{\circ}$ which related to the boundaries between Ferrel and Polar cells (Peixoto and Oort 92). The southern hemisphere contributions are larger than those of the northern hemisphere. The lack of symmetry with respect to the equator, on the strength of hemispheric contributions to the global AAM may depict the oceanic character of the southern hemisphere which makes zonal circulation stronger compared to the more continental northern hemisphere.

\section{Warming and $A A M$ trend}

The results plotted in Fig. 4 highlight the tropical trend values shown in Fig. 3; tropical peaks originate preferentially from high altitudes over the equatorial Tropopause $(6-23 \mathrm{~km},(400-30 \mathrm{hPa})$ but also from weaker amplitude contributions over the lower troposphere, i.e. from 1-4 km (900-600 hPa). Due to the near cancellation of negative and positive southern hemisphere contributions (Fig. 3), the prominent contribution seen about $10-25 \mathrm{~km}$ in Fig. 2 originates mostly from the equatorial bands. This structure agrees with the results by Satoh and Yoshida (1996). We remark similar responses in the equatorial upper troposphere and lower stratosphere although it is not clear whether these are directly related to the equatorial jet responding to the localized warming shown by Satoh and Yoshida (1996). This part of the lower stratosphere may also be influenced by the lowest region of the stratospheric Quasi-Biennial Oscillation (QBO). The interaction between a global warming and the response of the QBO has not yet been studied, mostly because until recently it has been very difficult to simulate QBO in global circulation models (Takahashi, 1996). This interaction should also be investigated.

\section{Summary and conclusion}

This study based on the AAM series computed with the zonal wind field from NCAR/NCEP reanalyses shows that AAM present a measurable and clear positive trend over the last 49 years (1949-1997). It is equivalent to a secular trend of $+0.56 \mathrm{~ms} / \mathrm{cy}$ in length of day. The trend in AAM is confirmed by four independent data sets arising from different atmospheric operational centers, i.e. ECMWF, UKMO, JMA and the NMC (now 


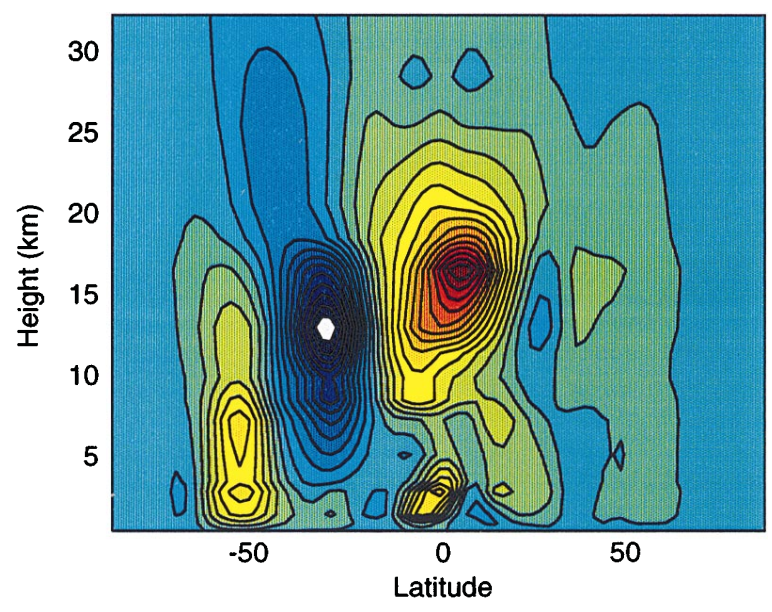

Fig. 4. Latitude-height distribution of contributions to the global AAM trend. The ordinate is height in kilometers $(\mathrm{km})$. Positive contributions are colored from green to red, negative contributions from blue to white

NCEP). Over the last 49 years, the computed trend in global (land + ocean) surface temperature is $0.79^{\circ} \mathrm{C} / \mathrm{cy}$. Consequently an increase of $0.1{ }^{\circ} \mathrm{C}$ per decade in global surface temperature will increase the global AAM by $0.07 \mathrm{~ms}$.

This study addresses the relative contributions of different parts of atmospheric angular momentum on the linear trend in LOD. It appears that the atmosphere as a whole gained angular momentum. The main contribution arises over the troposphere with almost $80 \%$ of the total trend in AAM, but the participation of the stratosphere is also important in terms of geographical contribution. The main contribution to the global AAM increase is located at the equator, one part near the surface but mostly within the equatorial Tropopause. This is consistent with a recent study by Satoh and Yoshida (1996) with an aqua planet model under global warming characteristics. Positive and negative values of trend are found within boundary zones of different atmospheric cells; they are positive between the Polar and Ferrel cells, and negative between the Hadley and Ferrel cells with particularly strong values associated within the main jet of each hemisphere. These results highlights the role played by the different cells (Hadley, Ferrel and Polar) and their boundaries on atmospheric angular momentum under global warming conditions. The values of the trend are generally higher over the southern hemisphere reflecting probably the larger ocean surface area.

Considering that changes in AAM for the entire atmosphere are accompanied by equal but opposite changes in the axial angular momentum of the solid earth, the value in LOD due to the AAM trend found $(+0.56 \mathrm{~ms} / \mathrm{cy})$ represent about the same value, though in the opposite sense, of contribution to trend in LOD measured by Laser ranging techniques $(-0.4$ to $-0.6 \mathrm{~ms} /$ cy) and attributed to postglacial rebound (Peltier and Jiang, 1996). The atmosphere is thus playing a major role at secular time-scales in LOD variability.
Since both contributions tend to cancel each other out, the actual (this century) trend in LOD must be close the secular trend due to tidal friction $(+2.3 \mathrm{~ms} /$ cy). Nevertheless, the observed secular increase in LOD $(+1.7 \mathrm{~ms} / \mathrm{cy})$ given by Stephenson and Morrison (1995) is obtained from all reliable historical observations over the past 2700 years (700 BC to AD 1900). Instead, the secular trend due to AAM is obtained exclusively from atmospheric reanalyses over the last 50 years. However, other components of the system such as oceanic circulation may be also participating actively at secular time scales, and their contribution will have to be carefully estimated before any definitive conclusion.

While the record of surface air (land and marine) temperature has for long been the principal evidence for change in the global system, atmospheric angular momentum, used until now to characterize the general circulation of the atmosphere, may be as well considered as a global warming indicator. Since the total angular momentum of the atmosphere-earth system is conserved the rotation rate of the solid Earth appears to be an indirect indicator. Furthermore this study questions whether an important effect of global warming, under an anthropogenic hypothesis, is the changing of the orbital characteristics of our planet.

Acknowledgements. We are grateful for discussion with Mme. Martine Feissel and comments by Mr. Masaki Satoh. Both provided critical readings of the manuscript and made several helpful and useful suggestions. An anonymous reviewer offered accurate and fruitful comments. Rosemary Morrow kindly assisted us in improving the English of this article. Special thanks to Peter Nelson from SBA (AER inc., Boston, USA) of International Earth Rotation Service (IERS) for providing us with the operational AAM data. We thank CPC/NCEP for providing us with the zonal wind fields from reanalyses of NCEP/NCAR.

Topical Editor F. Vial thanks S. Yoshida for his help in evaluating this paper.

\section{References}

Abarca del Rio, R., La Rotation de la Terre: Etude du cycle annuel et de la variabilité basse frequence. Etude climatique de la rotation Terrestre Ph.D. thesis, Université Paul Sabatier, 1997.

Barnes, R. T. H., R. Hide, A. A. White, and C. A. Wilson, Atmospheric angular momentum fluctuations, length of day changes and polar motion, Proc. R. Soc. Lon., 387, 31-73, 1983.

Christodoulis, D. C., D. E. Smith, R. G. Williamson, and S. M. Klosko, Observed tidal braking in the Earth/Moon/Sun system, J. Geophys. Res., 93, 6216-6236, 1988.

Eubanks, T. M., Variations in the orientation of the Earth, in Contributions of space geodesy to geodynamics, ed. by S. D. E., and D. L. Turcotte, American Geophysical Union, Washington, D.C., 1-54, 1993.

Kalnay, E., M. Kanahitsu, R. Kistler, W. Collins, D. Deaven, L. Gandin, H. Iredell, S. Saha, S. White, J. Woollen, Y. Zhu, M. Chelliah, W. Ebisuzaki, W. Higgins, J. Jandwiak, K. C. Mo, C. Ropelewski, J. Wang, A. Leetma, R. Reynolds, R. Jenne and D. Joseph, The NCEP/NCAR 40-year reanalysis project, Bull. Amer. Meteor. Soc., 77, 437-471, 1996.

Lambeck, K., The Earth's variable rotation: geophysical causes and consequences, Cambridge Univ. Press, Cambridge, 1980.

Lambeck, K., Geophysical geodesy: the slow deformation of the Earth, Clarendon Press, Oxford, 1988. 
Munk, W. H., and J. F. MacDonald, The rotation of the Earth, a geophysical discussion, Cambridge Univ. Press, Cambridge, 1960.

Nicholls, N., G. V. Gruza, V. Jouzel, T. R. Karl, L. A. Ogallo, and D. E. Parker, Observed climate variability, in The science of climatic change, ed. by Cambridge Univ. Press, Cambridge, 133-192, 1996.

Parker, D. E., P. D. Jones, C. K. Folland, and A. Bevan, Interdecadal changes of surface temperature since the late nineteenth century, J. Geophys. Res., 99, 14373-14399, 1994.

Peixoto, J. P., and A. H. Oort, Physics of Climate, American Institute of Physics, 1992.

Peltier, W. R., and X. Jiang, Glacial isostatic adjustment and Earth rotation: refined constraints on viscosity of the deepest mantle., J. Geophys. Res., 101, 3269-3290, 1996.

Rosen, R. D., and D. A. Salstein, Variations in atmospheric angular momentum on global and regional scales and length of day, $J$. Geophys. Res., 88, 5451-5470, 1983.
Salstein, D. A., D. M. Kann, A. J. Miller, and R. D. Rosen, The subbureau for atmospheric angular momentum of the international earth rotation service: a meteorological data center with geodetic applications, Bull. Amer. Meteor. Soc., 14, 67-80, 1993.

Satoh, M., and S. Yoshida, Response of the atmospheric angular momentum and the length of the day to the surface temperature increase for an aqua planet model, Geophys. Res. Lett., 23, 2569-2572, 1996.

Stefanick, M., Interannual atmospheric angular momentum variability 1963-1973 and the Southern Oscillation, J. Geophys. Res., 87, 428-432, 1982.

Stephenson, F. R., and L. V. Morrison, Long-term fluctuations in the Earth's rotation: 700 BC to AD 1990, Phil. Trans. R. Soc. A, 351, 165-202, 1995.

Takahashi, M., Simulation of the stratospheric quasi-biennial oscillation using a general circulation model, Geophys. Res. Lett., 23, 661-664, 1996

Wahr, J. M., The Earth's rotation, Ann. Rev. Earth Planet. Sci., 16, 231-249, 1988. 